\title{
RDUS
}

Revue de DROIT UNIVERSITÉ DE SHERBROOKE

Titre :

Auteur(s): $\quad$ Yves-Marie MORISSETTE

Revue: $\quad$ RDUS, 1999-2000, volume 30, numéro 1

Pages: $\quad 1-31$

ISSN : $\quad$ 0317-9656

Éditeur : $\quad$ Université de Sherbrooke. Faculté de droit.

URI : $\quad$ http://hdl.handle.net/1 1143/12353

DOI : $\quad$ https://doi.org/10.17118/11143/12353 
Page vide laissée intentionnellement. 


\section{FIGURE ACTUELLE DU JUGE DANS LA CITÉ}

par Yves-Marie MORISSETTE*

L'Association canadienne des professeurs de droit et l'Association canadienne droit et société ont choisi pour thème de la première rencontre de ce congrès Judges and the Public Space, expression qu'on a traduite par «Le rôle de la magistrature au sein de la société». Ce thème de discussion est tellement vaste, et tellement vague, qu'on pourrait l'aborder de toutes sortes de façons : la légitimité du juge, la transformation et, disent certains de nos jours, la politisation de la fonction judiciaire, la perception de la justice par le public, les rapports entre les tribunaux et les médias, etc.... etc.... En somme, on pourrait parler de tout et de rien sans jamais sortir du sujet. Afin d'éviter cela, il faut d'abord tenter de circonscrire ce thème.

Certains participants se pencheront plus spécifiquement sur ce que nous apprennent à ce sujet les Principes de déontologie judiciaire récemment publiés par le Conseil canadien de la magistrature. ${ }^{1}$ J'ai moi aussi quelques observations à livrer sur les Principes en question, mais je ne veux pas en faire le principal objet de mon intervention. Je vais plutôt tenter de faire deux choses : (I) dégrossir et analyser ce thème à la lumière de quelques distinctions qui me semblent pertinentes pour comprendre la situation du juge ici aujourd'hui, et (II) de façon plus spéculative, évoquer les raisons pour lesquelles le rôle du juge au Canada se transforme, et pourquoi ce rôle, dans ce qu'il a de plus essentiel, continue d'être peu ou mal compris. Je ne suis pas convaincu, d'ailleurs, qu'il soit souhaitable de remédier à cette relative incompréhension, car on peut se demander au sujet du juge, comme on a pu se le demander autrefois au sujet d'autres figures symboliques de la vie en société, s'il est vraiment opportun de semer le doute dans l'esprit des fidèles.

*. De la Faculté de droit et de l'Institut de droit comparé de l'Université McGill. Tout en assumant l'entière responsabilité du texte livré ici, l'auteur tient à remercier amicalement son collègue le $\mathrm{P}^{\mathrm{r}}$ Richard Janda pour les suggestions et commentaires lucides qu'il lui a faits. Ce texte est une version légèrement remaniée de la conférence prononcée le 6 juin 1999 à Sherbrooke.

1. Conseil canadien de la magistrature, Principes de déontologie judiciaire Ottawa, 1998. 


\section{- I -}

Mais commençons par explorer le contour du sujet avec quelques questions et distinctions simples. Je suppose, tout au long de cet exposé, que la société qui nous intéresse est celle que dessert le système de droit canadien, mais je me permettrai ici et là quelques comparaisons avec l'extérieur.

Première question : de quels juges s'agit-il au juste? De juges de première instance ou de juges d'appel, de juges à temps partiel, comme la plupart des juges de cours municipales ou de juges de carrière qui donnent tout leur temps à leurs fonctions, de juges du droit commun, interprètes dans la plupart des cas (du moins théoriquement) de l'intention du législateur, ou de juges constitutionnels, pour qui (théoriquement) l'intention du constituant a préséance presque tous les jours sur celle du législateur. Je sais que ces catégories sont imparfaites et se recoupent. Il n'y a pas ici, comme en Allemagne, en France ou en Autriche, un tribunal dont la fonction exclusive est de trancher les litiges constitutionnels, mais la Cour suprême du Canada, comparativement parlant, passe plus de temps que tous les autres tribunaux canadiens sur les questions de cet ordre. À l'opposé, la notion même de «tribunal de première instance» prête à interprétation : ainsi les cours supérieures deviennent en quelque sorte des tribunaux d'appel sur le droit lorsqu'elles exercent leur compétence dans le champ étendu et important du contentieux administratif.

Néanmoins, ces distinctions me paraissent nécessaires, car le pouvoir judiciaire n'est pas un monolithe compact et tous les juges ne participent pas avec une égale assiduité à l'entreprise d'édification normative dans la société. Certains, principalement les juges de première instance, passent une partie importante de leur temps à la mise en oeuvre de normes plutôt claires et plutôt stables dans des cas particuliers (ils appartiennent à un appareil de sanction du droit et, selon la distinction classique de H.L.A. Hart, ${ }^{2}$ vivent près du noyau dur

2. Il s'agit, évidemment, de la distinction qui ressort d'un pan du célèbre débat entre H.L.A. Hart et Lon Fuller : voir H.L.A. Hart, «Positivism and the Separation of Law and Morals» (1958), 71 Harv. L. Rev. 593 aux pp. 606-615, et Lon Fuller, «Positivism and Fidelity to Law - A Reply to Professor Hart» (1958), 71 Harv. L. Rev. 630 aux pp. 661-669. Il est hors de question d'entrer ici dans ce débat et de considérer la thèse de Hart, développée plus 
- the core - du droit); d'autres, principalement les juges des juridictions d'appel, interviennent là où le droit est indéterminé (ils appartiennent à un appareil de création ou d'invention du droit et vivent dans le halo - the penumbra - du droit). On peut avancer de façon générale que la distinction entre le sommet et la base de la hiérarchie judiciaire correspond à cette répartition des fonctions et qu'elle a une réelle pertinence ici.

Je ne veux pas laisser entendre par là que les juges de première instance sont nécessairement et complètement absent de l'espace public : il est évident que ce n'est pas le cas. Certains procès à grand retentissement font les manchettes et occupent l'attention du public pendant des semaines : le procès de Paul Bernardo en est un exemple. En outre, certaines décisions de première instance qui passeraient normalement inaperçues ont parfois pour effet de propulser leurs auteurs sur la place publique, à la première page des journaux : je pense ici aux déboires des juges Raymonde Verreault ou Monique Dubreuil au Québec. ${ }^{3}$ La plupart du temps, cependant, ces incidents, et même les cas très controversés qui appellent des sanctions contre le juge, comme celui des juges Jean Bienvenue, René Crochetière ou Denys Dionne, sont sans conséquence durable. Du point de vue du corps constitué qu'est la magistrature, ce sont des aberrations qui, une fois identifiées comme telles, illustrent quel genre de conduite est inacceptable aujourd'hui. Du point de vue de la société en général, ce sont des faits divers qui s'estompent dans le bruit de fond permanent sur la justice et l'activité des tribunaux.

Dans les dossiers les plus importants, ceux dont on peut dire, pour user d'une expression éculée, qu'ils comportent des «enjeux de société», il est rare

avant dans The Concept of Law $2^{\mathrm{e}}$ éd., Oxford, Clarendon Press, 1961à la p. 127 et s. Je me contenterai de dire pour la compréhension de ce qui suit que, sur le problème de l'indétermination en droit, je me range du côté de Hart, tout en acceptant que sa thèse n'explique pas tout.

3. L'une et l'autre, en 1994 et en 1998 respectivement, ont été exonérées par le Conseil de la magistrature. Ces deux cas font partie du sous-ensemble croissant des affaires où l'on porte devant un conseil de la magistrature, ou un autre organisme semblable, des situations qui devraient relever des tribunaux d'appel. On voit donc de manière insinuante se répandre l'idée saugrenue que le juge qui aurait erré en droit, selon l'euphémisme usuel, aurait aussi fauté professionnellement. Fâcheuse baliverne. Pourquoi aussi ne pas tenir les juges civilement responsables des erreurs pourtant extrêmement coûteuses qu'ils commettent chaque fois qu'une cour d'appel leur donne tort? 
qu'un juge de première instance soit l'objet d'une controverse publique prolongée. Et c'est tant mieux. La raison en est simple : si la question en cause revêt véritablement ce genre d'importance, elle fera son chemin jusqu'aux tribunaux d'appel. Il y a, bien sûr, des exceptions. Je pense ici à un cas récent : celui du juge John Reilly de la Cour provinciale d'Alberta entré en conflit avec le juge en chef et le juge en chef adjoint de sa juridiction sur une question de principe relative au traitement des justiciables autochtones. ${ }^{4}$ À ma connaissance, de tels exemples sont très inhabituels.

En fin de compte, je continue de croire que, comme je l'ai déjà écrit en $1994,{ }^{5}$ lorsque l'on considère l'appareil judiciaire dans son ensemble, il y a un parallèle à faire avec l'exécutif : la notoriété du cabinet et l'anonymat de la fonction publique se retrouvent aussi dans la magistrature.

La publicité intense qui entoure désormais les nominations à la Cour suprême du Canada, et qui dépasse tout ce que l'on a connu au niveau des cours d'appel, en est un indice. Cela explique aussi les multiples références à ce tribunal dans la grande presse, références désormais presque quasi-quotidiennes entre septembre et juin. Il se peut que ce phénomène ait un effet d'entraînement. Ainsi, le journaliste Gordon Gibson commençait en ces termes dans les pages éditoriales du Globe and Mail un article récent qui pourtant était entièrement consacré à la Cour suprême :

«Nowadays there is more newsworthy action year-round in the courts than in the federal and provincial legislatures taken together. That does not mean, alas, that governments have stopped interfering with our lives. It means the courts are busier than ever making decisions on public policy.» ${ }^{6}$

4. Les circonstances de cette affaire sont relatées en détail dans le jugement Reilly c. Alberta (Provincial Court, Chief Judge) [1999] A.J. n 464, en ligne : QL (WWR).

5. Voir Y.-M. Morissette, «Le processus de nomination des juges en regard de leur "immense pouvoir politique"» (1994), 25 R.G.D. 307 à la p. 309, note 13.

6. G. Gibson, «What the Supreme Court has to say about it», Globe and Mail, (1 $1^{\mathrm{e}}$ juin 1999) A-15. 
Deuxième question : le juge, oui, mais en quelle qualité? En sa qualité de serviteur du droit, de dispensateur de la justice, ou en sa qualité de sujet de droit? En sa qualité d'arbitre du débat ou de partie au débat? En tant que présence passive ou active dans la société?

Cette question est peut-être moins évidente que la première, mais elle acquiert depuis quelque temps de plus en plus de pertinence. Elle me paraît un autre révélateur de la transformation qu'a subi la magistrature au cours des vingt dernières années.

Il n'y a pas si longtemps, la personne du juge s'effaçait entièrement ou presque derrière son rôle : en accédant à la magistrature, le juge quittait en quelque sorte le champ de bataille des droits pour monter sur les hauteurs. La transformation était irréversible. On raconte par exemple que lorsque F.E. Smith fut promu directement, à l'âge de 46 ans, au plus haut échelon de la magistrature britannique, il le fit avec une certaine mélancolie, et non sans inquiétude, car il renonçait une fois pour toutes à une carrière étincelante de barrister. ${ }^{7}$ De nos jours, le retour à la pratique après la magistrature est chose courante. ${ }^{8}$ On peut bien sûr se gausser de ces choses-là, surtout lorsque l'on a une foi inébranlable dans le progrès et que l'on croit dur comme fer que l'écoulement du temps est le synonyme exact et linéaire de l'avancement de la condition humaine. Pour ma part, je crois que ces attitudes aujourd'hui désuètes des juges anglais étaient empreintes d'une réelle noblesse. L'accession à la magistrature, et au redoutable pouvoir de juger ses semblables, ne se concevait pas sans un renoncement aux choses de ce monde. Ce n'est plus autant le cas aujourd'hui.

7. Voir R.F.V. Heuston, Lives of the Lord Chancellors 1885-1940, Oxford, Clarendon Press, 1964 à la p. 381 : «It cut him off from the prospect of further political advancement and also from the chance of returning to the Bar. It, also perhaps more seriously, curtailed his income by a considerable degree.» Voir aussi F.W.F. Smith, The life of F.E. Smith, first Earl of Birkenhead, London, Eyre \& Spottiswoode, 1960 à la p. 380 : «Acceptance of the Woolsack meant that F.E.Smith could never resume his practice at the Bar. If and when the Coalition Ministry came to an end, and he had to retire from his office, he must be content with a Lord Chancellor's pension and with the duty that devolves upon an ex-Lord Chancellor of assisting in the judicial work of the House of Lords.»

8. Bien entendu, il faut tenir compte du fait que, sauf pour le Lord Chancellor dans cette fonction, les juges des juridictions supérieures étaient nommés à vie. Il existe encore aujourd'hui au Canada des restrictions légales sur le droit de pratique des anciens juges; elles sont mentionnées par le juge Sopinka dans le texte cité plus loin, infra note 18 à la p. 173. 
Surtout, le juge n'est plus absent du droit en tant que sujet de droit. Lui aussi a des droits, et il les revendique, ce qui est un phénomène assez nouveau. Avant les années '90, le droit canadien ne porte presque aucune trace du juge en tant que détenteur de droits. Dans les rares $\operatorname{cas}^{9}$ où un juge se manifestait de la sorte, ses interventions passaient à peu près inaperçues en raison de leur caractère essentiellement technique et relatif à l'administration de la justice : elles n'intéressaient que les juristes (voire certains d'entre eux seulement) et il était moins question des droits du juge que des attributs s'attachant à l'indépendance judiciaire. ${ }^{10}$ Le cas considérablement plus controversé du juge Thomas Berger ne fait pas exception à la règle : à mon sens, il la confirme. Les circonstances de cette affaire sont connues. ${ }^{11}$ On peut en retenir que, même si le juge Berger a choisi de démissionner après avoir essuyé une réprimande, il n'a pas tenté de justifier sa conduite revendiquant son droit à la liberté d'expression en tant que citoyen. ${ }^{12}$

L'idée, cependant, que le juge puisse invoquer un droit de parole, ou un droit à la liberté d'expression, progresse au Canada. Ici encore, il y a une convergence apparente avec le droit américain. La ressemblance n'est toutefois que partielle : n'oublions pas que (1) la grande majorité des juges des états américains sont élus ou doivent sporadiquement se soumettre à un processus électoral après avoir été nommés ${ }^{13}$ (je ne parle pas ici des juges de nomination

9. Je pense ici à des affaires comme R. c. Beauregard, [1986] 2 R.C.S. 56 ou Landreville c. R., [1977] 1 C.F. 419, Landreville c. R. (No. 2), [1977] 2 C.F. 726 et Landreville c. R. (No. 3), [1981] 1 C.F. 15.

10. Un exemple récent et beaucoup plus ambitieux de ce genre de raisonnement : le Renvoi sur la rémunération des juges de la Cour provinciale de l'Île-du-Prince-Édouard, [1997] 3 R.C.S. 3.

11. Elle sont notamment passées en revue par Jeremy Webber. J. Webber, «The Limits to Judges' Free Speech : A Comment on the Report of the Committee of Investigation into the Conduct of the Hon. Mr Justice Berger» (1983-1984), 29 McGill L.J. 369.

12. Les documents pertinents sont reproduits en annexe au rapport publiés «Report and Record of the Committee of Investigation into the Conduct of the Hon. Mr Justice Berger and Resolution of the Canadian Judicial Council» (1983), 28 McGill L.J. 378. Notons que, selon le juge Berger, trois exemples de conduite comparable à la sienne, par le juge Thorson, le juge en chef Freedman et Lord Denning, démontraient que son cas n'était pas sans précédent. Ibid. à la p. 404.

13. Voir sur cette question S. P. Croley, «The Majoritarian Difficulty : Elective Judiciairies and the Rule of Law» (1995), 62 U. Chi. L. Rev. 689 aux pp. 725-726. 
fédérale), (2) les juges de nomination fédérale doivent tous se soumettre au processus des confirmatory hearings, qui n'est pas également éprouvant pour tous, mais qui l'est parfois singulièrement, comme ce fut le cas du juge Clarence Thomas, ${ }^{14}$ et (3) bien entendu, il y a en droit américain une culture très vivace de la liberté d'expression, fondée sur le Ier Amendement. Là plus qu'ailleurs, par conséquent, les juges ont besoin d'avoir les coudées franches lorsqu'ils s'expriment en public. Et ils ont les moyens juridiques de s'en assurer. Aussi la question n'est-elle pas nouvelle aux États-Unis, où elle a souvent été débattue devant les tribunaux. ${ }^{15}$

Au Canada, le débat a été lancé sous cet angle (c'est-à-dire celui du droit individuel du juge à la liberté d'expression) en 1995, dans le sillage de l'affaire Angers. Je rappelle qu'à cette occasion un comité du Conseil canadien de la magistrature avait sévèrement critiqué ${ }^{16}$ un juge de la Cour d'appel du NouveauBrunswick parce qu'il avait adressé au premier ministre du Canada et aux

14. C'est ce qui fait dire au sociologue américain Seymour Lipset, spécialiste des comparaisons entre le Canada et les États-Unis, «[...] the American system now makes the number one criterion for a Supreme Court nomination, confirmability. A recent chief justice told me he had spent 15 minutes with the president who appointed him and had never met him before. It is now almost impossible for strong conservatives or liberals to get confirmed.» S. Lipset, «Parlimentary Review of Supreme Court Appointments Questioned», 1998, en ligne : Fraser Forum Online, Reader Response, $<\mathrm{http}: / / \mathrm{www}$. fraserinstitute.ca/publications/ forum/1998/may/your_turn.html> (date d'accès 25 novembre 1999). Pour un exemple assez frappant de judicial free speech, où un juge s'en prend à un autre d'une manière qualifiée ailleurs de patronizing et insulting (voir J. C. Kelso, «Time, Place, and Manner Restrictions on Extrajudicial Speech by Judges» (1995), 28 Loy. L.A. L. Rev. 851 à la p. 867), consulter A. L. Higginbotham, «An Open Letter to Justice Clarence Thomas from a Federal Judicial Colleague» (1992), 140 U. Pa. L. Rev. aux pp. 1005-1028.

15. On cite fréquemment à ce sujet l'article de T. D'Alemberte, «Searching for the Limits of Judicial Free Speech»(1987), 61 Tul. L. Rev. 611, mais voir aussi le numéro spécial «Symposium : The Sound of the Gavel : Perspectives on Judicial Speech» (1995), 28 Loy. L.A. L. Rev. à la p.795 et s. Dans la plupart des états américains, on trouve des dispositions relatives à cette forme d'expression dans les Codes of Judicial Conduct; cela suscite inévitablement des questions au regard du I ${ }^{\text {er }}$ Amendement. Voir E. Chemerinsky, «Is it the Siren Call? Judges and Free Speech While Cases are Pending», Loy. L.A. Rev. 831, à la p. 840, où est considérée l'affaire Scott v. Flowers, 910 F.2d 201 (5th Cir. 1990), et voir enfin R. O'Neil, «Assaults on the Judiciairy» (1998), 34-SEP Trial 54, pour un point de vue franchement hostile aux restrictions à la liberté d'expression.

16. «We strongly disapprove your conduct...», conclut le rapport, reproduit in extenso avec la lettre litigieuse dans la revue citée infra, note 17 à la p. 183 et s. 
membres du Parlement une lettre ouverte contestant vigoureusement la politique du gouvernement canadien sur le contrôle des armes à feu. De cette passe d'armes, si l'on peut dire, est sorti un colloque universitaire dont les travaux furent publiés dans la Revue de droit de l'Université du NouveauBrunswick. ${ }^{17}$ On y trouve entre autres, sur la question de la liberté d'expression judiciaire, des communications de spécialistes des questions d'administration de la justice (Peter Russell ${ }^{18}$ et Beverley Smith ${ }^{19}$ ) ainsi que des textes du juge John Sopinka ${ }^{20}$ et du professeur Jacob Ziegel. ${ }^{21}$

Quelques mois après l'affaire Angers, la Cour suprême rendait jugement dans le dossier Ruffo c. Conseil de la magistrature, ${ }^{22}$ où l'appelante avait plaidé l'inconstitutionnalité de l'article 8 du Code de déontologie de la magistrature du Québec ${ }^{23}$ pour violation de l'alinéa 2 b) de la Charte canadienne des droits et libertés. Le jugement majoritaire de la Cour, rédigé par le juge Gonthier et qui porte principalement sur des considérations d'impartialité institutionnelle relatives au Conseil de la magistrature du Québec, conclut (1) que l'article 8, lequel restreint la liberté d'expression, n'est pas inconstitutionnel pour cause d'imprécision, mais (2) qu'il est prématuré de trancher la question de savoir si

17. «Judicial Free Speech : Forum» (1996), 45 R.D.U.N.-B. aux pp. 77-189.

18. «Judicial Free Speech : Justifiable Limits», ibid. à la p. 155.

19. «Judicial Free Speech in Canada», ibid. à la p.161. On constate par les sources qu'utilise l'auteur que la question est nouvelle en droit canadien.

20. «Must a Judge be a Monk - Revisited», ibid. à la p. 167. Il s'agit d'une version substantiellement remaniée d'un discours prononcé en 1989 devant l'Association du Barreau canadien. Le juge Sopinka fait état de quatre plaintes reçues par le Conseil canadien de la magistrature pour des prises de position publiques par des juges. Aucune n'a mené à une destitution. Deux ont engendré une réprimande (les juges Berger et Angers), deux ont été jugées sans fondement (les juges B. Wilson et McLachlin).

21. «Judicial Free Speech and Judicial Accountability : Striking the Right Balance», ibid. à la p. 175.

22. Ruffo c. Conseil de la magistrature, [1995] 4 R.C.S. 267 [ci-après Ruffo].

23. 114 G.O.Q.1982.II.1648. L'article en question se lit : «Dans son comportement public le juge doit faire preuve de réserve, de courtoisie et de sérénité.» Je note que dans l'article précité, supra note 16 à la p. 159, le professeur Russell émet des doutes sur l'opportunité d'une telle disposition, du moins en ce qui concerne la réserve et la sérénité. Je reviendrai sur ce point mais je signale en passant que nous avons probablement ici un exemple des méfaits d'une traduction approximative : reserve en anglais (terme utilisé dans la version anglaise du Code) n'a pas la connotation que «devoir de réserve» a immédiatement en français. 
ce même article contrevient à l'alinéa 2b) de la Charte. ${ }^{24}$ Dans l'ordre du droit positif, donc, la question des limites permises à la liberté d'expression du juge canadien demeure ouverte, ou en tout cas pas complètement tranchée.

Il en résulte pour le juge canadien un modus vivendi : il doit s'abstenir de prendre publiquement position sur une question politiquement controversée, et la rigueur de cette obligation de réserve augmente en fonction du risque que la question donne naissance à un litige dont le juge pourrait être saisi. ${ }^{25}$ Il a cependant le droit de prendre publiquement position, de manière non partisane et avec mesure, sur des questions relatives à l'administration de la justice, y compris des questions juridiques d'intérêt général. La tendance est vers l'ouverture ${ }^{26}$ et ces restrictions, moins strictes qu'autrefois, s'apparentent à ce que l'on connaît aux États-Unis. À mon sens, l'effet de cette plus grande

24. Infra, En réponse à la question «l'article 8 [...] est-il contraire à l'al. 2b) [...] ?», la Cour répond, dans le dispositif du jugement : «Il est prématuré [...] de se prononcer à ce stade sur la question telle que formulée. Cependant, le devoir de réserve n'est pas jugé inconstitutionnel pour cause d'imprécision.» (par. 113 du jugement). Il faut donc supposer que cet article 8 constitue une «règle de droit» au sens de l'article 1 de la Charte, mais pas nécessairement une limite raisonnable dont la justification est démontrable, nuance qui ressort clairement du jugement dissident du juge Sopinka, d'accord avec son collègue sur ce point (par. 115 du jugement).

25. Un passage de la lettre adressée au juge Angers par le comité d'enquête du Conseil canadien de la magistrature est révélateur à cet égard : «We pause to say, that unlike Mr. Justice Berger, who was commenting on matters of high constitutional importance that would rarely come before him as a judge, yours is a highly partisan attack upon a proposal which, if carried forward into legislation, could well come frequently before you for interpretation or enforcement.» Supra note 17 à la p. 187.

26. Il semble bien que les opinions hardies exprimées il y a près de vingt ans par le politologue Léon Dion dans un discours destiné aux membres de l'Association canadienne des juges de cours provinciales («Plus de démocratie pour les juges» (1981), 41 R. du B. 199) soient beaucoup plus près aujourd'hui de la pensée dominante véhiculée par le droit positif : voir par exemple les observations sur les juges dans la récente décision Ruffo c. Québec (Ministre de la Justice), [1998] R.J.Q. 254 (C.S.). Le juge en chef Laskin, dont l'attitude générale n'était pourtant pas inamicale envers les droits individuels et les libertés civiles, voyait les choses de manière plus traditionnelle : «[a] judge has no freedom of speech to address political issues which have nothing to do with his judicial duties. His abstention from political involvement is one of the guarantees of his impartiality, his integrity, his independence...» («Berger and Free Speech of the Judge» allocution pour l'Association du Barreau canadien le 2 spetembre 1982, citée dans W. MacKay, «Judicial Free Speech and Accontability : Should Judges Be Seen but Not Heard?» (1993), 3 N.J.C.L. 159 à la p. 213. «His indeed» diraient sans doute certain(e)s de nos jours. 
ouverture est le suivant : les propos qui dénotent un manque d'impartialité apparent continueront d'être sanctionnés par les moyens de droit habituels (demande de récusation et, à la limite, sanctions disciplinaires), mais rien n'empêchera plus un juge qui manque de jugement de faire publiquement étalage de cette lacune, il faut bien le dire, assez préoccupante chez un juge. Nous en sommes déjà là, à mon avis : c'est la question que pose, péniblement et interminablement, l'affaire Ruffo.

Concédons que le juge soit, comme tout le monde, un sujet de droit. Son statut lui impose cependant, au risque de le discréditer et même de jeter le discrédit sur ses collègues, qu'il se montre plus responsable que d'autres dans l'exercice de ses droits. C'est, je crois, l'idée qui sourd dans un article récent du journaliste Yves Boisvert sur le déroulement de l'affaire Flahiff, article qu'il a intitulé plaisamment Le niaisage déontologique. ${ }^{27}$

Troisième question : $\mathbf{y}$ a-t-il un espace privé pour le juge comme pour ses concitoyens? Les juges sont aussi des êtres humains. En tant que spécimen anthropologique, le juge a une vie privée, il peut collectionner les armes à feu et priser la chasse, divorcer, faire faillite, souffrir d'une dépression nerveuse, être atteint d'une toxicomanie, avoir pendant qu'il est juge, avant de le devenir ou après l'avoir été, des comportements qui le contraindront à démissionner (comme le juge Flahiff, qui à cet égard est sur le même pied que ses concitoyens), dont la dissimulation provoqueront sa destitution (comme le juge Therrien qui, à cet égard, n'est pas sur le même pied que ses concitoyens) ou qui lui vaudront d'être jugé plus sévèrement que ses concitoyens même après avoir quitté la magistrature. ${ }^{28}$ Il y a des indiscrétions et des incartades, parfois

27. La Presse (10 avril 1999) p. B-3.

28. Une dépêche de la Presse Canadienne en date du 10 mai 1995 relate ce qui suit : «Un ancien magistrat a été accusé hier de vol à l'étalage, recel et corruption de fonctionnaire. Âgé de 79 ans, l'ex-juge de la cour provinciale [...] aurait été pincé le 15 avril dernier au Marché Jimmy, une épicerie de Knowlton, en possession d'un filet de boeuf - un steak de $8 \$$ - qu'il aurait chipé. Or à l'agent de police venu l'interpeller formellement, l'ex-magistrat aurait offert de l'argent, question d'éviter les conséquences fâcheuses de son geste. Le policier ne s'est toutefois pas laissé soudoyer. Trouvé coupable d'un vol à l'étalage, le commun des mortels s'en tire habituellement avec une amende variant entre $100 \$$ et $200 \$$. L'accusation de recel n'est qu'accessoire. Cependant, la tentative de corruption d'un agent de la paix est un crime plus grave qui peut encourir une peine pouvant aller jusqu'à 14 ans de prison. Joint hier soir 
assez inoffensives, qui, dans le cas d'un juge, ne pardonnent pas. Ainsi, on a vu il y a quelques années un juge démissionner en raison d'un incident assez triste survenu lorsque sa conjointe et lui avaient été arrêtés à un barrage routier. ${ }^{29}$ Toute infraction commise par un juge, sauf peut-être les infractions routières les plus bénignes, risque d'être perçue comme un genre de forfaiture.

Certes, les juges ne sont pas les seuls dans cette situation. Je pense ici à l'épisode récent qui a provoqué la démission d'une présidente de centrale syndicale pour vol à l'étalage d'une paire de gants. Mais la vulnérabilité du juge est nettement plus grande que celle du commun des mortels, ou des «élites»en général : c'est un peu comme si sa fonction, qui consiste à juger autrui, lui imposait de se placer hors de portée du jugement d'autrui. On s'attend de sa part à un degré de discrétion, de décence et de mesure, dans sa vie privée comme dans sa vie publique, qui interdit la plupart des écarts de conduite ou qui, du moins, en rend nécessaire la dissimulation. ${ }^{30}$ Le juge ne peut être humain en public que sous un jour positif. Les choses ont évolué pour le juge comme pour

à son domicile, et appelé à commenter l'affaire, M. [...] a paru estomaqué. En peu de mots, il a mentionné qu'il s'agit d'une simple erreur. "Je la regrette infiniment et je suis très humilié", a-t-il dit.» Le juge en question était retraité depuis 1987.

. Voici la description qu'en donne une dépêche de la Presse Canadienne en date du 29 novembre 1996 : «Le juge [...] de la Cour supérieure a plaidé coupable à une accusation d'entrave au travail de policiers de la Sûreté du Québec (SQ) et, par la voie de son avocat, a fait savoir qu'il avait remis sa démission comme membre de la magistrature, hier, au palais de justice de Shawinigan, en Mauricie. Le juge Bernard Grenier, de la Cour du Québec du district de Montréal, a décidé de lui faire bénéficier d'une absolution conditionnelle [...]. En rappelant les événements ayant conduit le magistrat au banc des accusés, le procureur de la poursuite, Me Michel Breton, de Longueuil, a signalé que le 1er septembre dernier, à Baie-de-Shawinigan, le juge et son épouse, au volant du véhicule dans lequel ils voyageaient, s'étaient retrouvés à un barrage routier. Les policiers de la SQ, constatant l'odeur d'alcool se dégageant du véhicule, avaient soumis Mme [...] à un test d'alcoolémie. Le juge [...], "visiblement en état d'ébriété très avancée", selon les policiers, était intervenu, disant à son épouse de ne pas souffler dans l'appareil. Il avait ensuite laissé entendre aux policiers qu'entre gens du milieu de la justice, il fallait se protéger. Alors que l'on emmenait son épouse au poste de la SQ, pour y souffler dans un alcootest, le juge avait supplié à genoux les agents.»

30. On peut rappeler ici à titre d'exemple les habitudes de consommation d'alcool de Lyman Poore Duff (très certainement l'un des plus éminents juristes de l'histoire du Canada ainsi qu' un juge en chef respecté) dont son biographe fait état avec tact : voir D. R. Williams, Duff : a life in the law, Vancouver; University of British Columbia Press, en association avec Osgoode Society, 1984 (consulter l'index sous Duff, L.P., alcoholism). 
tout le monde, de sorte que, par exemple, un problème comme l'alcoolisme n'est plus aujourd'hui, autant qu'il l'était autrefois, une circonstance à taire ou à enfouir. Il existe maintenant pour la magistrature des programmes de soutien destinés à résoudre les difficultés de cet ordre et qui, avec un peu de bonne volonté, peuvent prendre le relais des pressions exercées sur la personne du juge par ses collègues. Le juge demeure néanmoins dans une catégorie à part et il peut souffrir plus que d'autres de la divulgation d'un renseignement de ce genre : étant donné la nature de sa fonction, le public réagirait-il dans son cas à la révélation d'une dépendance à l'alcool avec la même générosité dont il fit montre, par exemple, dans le cas de Mme Betty Ford, épouse de l'ancien président des États-Unis? Probablement pas. Il n'est pas déraisonnable de considérer la sobriété comme une préalable à l'exercice éclairé du jugement.

Il y a là un fait de société commun à la plupart, sinon à la totalité, des pays dotés d'un système de droit anglo-saxon. Cela tient à la place prééminente du juge dans cette culture politique. Offrons un contraste : peut-on imaginer un seul instant que les médias, la classe politique ou le public du Canada toléreraient de la part du juge en chef de la Cour suprême le genre de comportement qui, en France, a été toléré pendant plus d'un an et demi par le vis-à-vis fonctionnel du juge Lamer, M. Roland Dumas, président du Conseil constitutionnel français? ${ }^{31}$ Non.

31. Voir à ce sujet dans Le Monde du 6 mars 1999 l'article intitulé «La chronologie de l'affaire», en ligne : Le Monde édition électronique <http://wwwlemonde.fr/ article/0,2320,4566,00.html> (dernière modification : 6 mars 1999); voir aussi «Le conseil constitutionnel au péril de l'intérim», <http://www.lemonde.fr/ article/0,2320,4596,00.html> (dernière modification : 21 mai 1999). Les circonstances qui ont mené à la démission du juge Abe Fortas de la Cour suprême des États-Unis en 1969, et celles qui ont précédé la mort du juge Lionel Murphy de la High Court of Australia en 1987, confirment à mon sens plutôt qu'elles n'infirment le jugement qui est porté ici. 
- II -

La plupart des éléments que j'ai avancés jusqu'ici sont assez banals et font depuis longtemps partie du domaine public. Je voudrais maintenant pousser la réflexion un peu plus loin en abordant deux aspects : comment et pourquoi la fonction du juge se transforme-t-elle aujourd'hui au Canada? Dans quelle mesure la nature intime de cette fonction est-elle comprise du grand public?

Les Principes de déontologie judiciaire et le rapport entre le juge et la société. Le rapport entre le juge et la société où il officie est symbiotique ou interactif, dirait-on plutôt aujourd'hui. Juger à Bordeaux, à Téhéran ou à Hanoi, ce n'est décidément pas la même chose que juger à Sherbrooke ou à Moncton. Les universitaires québécois qui depuis quelques années participent à la formation de juges de la République populaire de Chine ou du Vietnam constatent rapidement que la compréhension du droit occidental présente généralement moins de difficulté pour ces magistrats que la compréhension du contexte social dans lequel le droit s'insère, de l'a priori collectif qui sert de toile de fond au droit.

La fonction du juge, et son image dans la cité, évoluent donc comme la société elle-même, et à peu près au même rythme qu'elle. Or, nous vivons actuellement au Canada une période où le concept d'égalité, et le programme égalitaire, sont envahissants. Si nous transplantions ici la devise de la République française, «Liberté, Égalité, Fraternité», nous pourrions avancer que l'époque de la liberté occupa la majeure partie des $\mathrm{XIX}^{\mathrm{e}}$ et $\mathrm{XX}^{\mathrm{e}}$ siècle, ${ }^{32}$ que celle de l'égalité a commencé avec l'après-guerre, et que celle de la fraternité (ou de la solidarité, dirait-on plus volontiers aujourd'hui) est encore devant nous.

D'autre part, les systèmes de valeurs qui nous ont soutenus pendant un siècle après la Confédération, et parmi lesquels je rangerais la religion et le conformisme social de la société industrielle et pré-industrielle, ont considérablement perdu de leur emprise depuis vingt ans.

32. J'entends par là l'époque du libéralisme débridé. Bien entendu, on peut soutenir que cette forme de liberté au profit des uns (par ex., le capital) brimait les autres (par ex., le prolétariat). 
Il s'ensuit plusieurs conséquences, comme par exemple la «demande d'éthique», un phénomène bien analysé par le sociologue Guy Rocher. ${ }^{33} \mathrm{Je}$ résume sa thèse sans, je l'espère, la déformer. On a vu au cours des deux ou trois dernières générations quatre facteurs lourds transformer les valeurs. D'abord, (I), la montée de la classe moyenne, avec un ensemble d'effets secondaires sur les valeurs. Par exemple, la quasi-disparition de la classe rurale ou l'hégémonie grandissante d'une mentalité individualiste (d'où les droits de la personne) et utilitariste (d'où le consumérisme envahissant et réducteur). Ensuite, (II), selon l'expression de Weber, le désenchantement du monde et de l'histoire : «...avec le progrès de la science et puis aujourd'hui de la technoscience, on a assisté à un recul et à un déclin des mythologies, des religions, des grandes certitudes, des fois et des valeurs antérieures. Dieu est mort scientifiquement...» ${ }^{34}$ Puis, (III), la mutation des rapports sociaux, avec entre autres rééquilibrages fondamentaux, celui des rapports entre les hommes et les femmes, et celui aussi des rapports entre ethnies, races et cultures différentes, qui aujourd'hui se côtoient partout dans les mêmes sociétés. «Cela fait surgir un nouveau thème éthique, dit Rocher, celui de la différence humaine.» ${ }^{35}$ Enfin, (IV), la fragmentation des sphères de la vie et de la culture. Un exemple que nous connaissons bien en tant que juristes : le droit, dans la société contemporaine, «s'est progressivement autonomisé, il s'est détaché de la morale, détaché des religions, détaché du politique.» Cela est vrai du droit, cela est vrai aussi d'une quantité d'autres choses : par exemple, production et consommation se sont fragmentées dans les ménages, nous ne sommes plus à l'époque où une famille vivait de ce qu'elle produisait sur la ferme. Il en va de même pour le phénomène de la professionnalisation de l'éthique : l'éthique n'est plus diffuse et partagée par tous, mais elle devient explicite (on l'enseigne) et elle est confié à des spécialistes (les comités d'éthique dans les hôpitaux où les centres de recherche, les comités de déontologie dans les professions, le Conseil de presse, les

33. Voir «Le défi éthique dans un contexte social et culturel en mutation» dans G. Rocher, Études de sociologie du droit et de l'éthique, Montréal, Thémis, 1996 dans pp. 305-317. Je reprends ici une argumentation déjà utilisée dans «Les fonctions respectives de la déontologie et de la justice naturelle dans la justice administrative», dans Y.-M. Morissette, Développements récents en droit administratif et constitutionnel, Cowansville, Service de la formation permanente du Barreau du Québec et Éditions Yvon Blais, 1999 à la p. 21 et s.

34. Ibid. à la p. 310.

35. Ibid. à la p. 312. 
«éthiciens», etc.). Cela fait dire à Rocher, et ce sera mon dernier emprunt : «Plus on sent et craint le vide moral et plus on sent la demande de morale, plus on risque d'être entraîné dans des excès de régulation qui se prennent pour de la vertu et pour de la pureté morale.» ${ }^{36}$ D'où la «demande d'éthique».

Ces facteurs conduisent à l'adoption de codes d'éthique dans toutes sortes de domaines. Les Principes de déontologie judiciaire, ${ }^{37}$ au sujet desquels je ferai deux courtes séries de remarques, s'inscrivent dans ce mouvement.

1. Sur la forme, je crois que ce document est très réussi et qu'il a évité plusieurs écueils. Il se présente comme un énoncé de principes de nature exhortatoire, ${ }^{38}$ accompagnés de commentaires qui parfois comprennent des questions laissées délibérément ouvertes. Des renvois à des sources externes, et en particulier jurisprudentielles, accentuent le caractère discursif et didactique de l'ensemble. Cette façon d'aborder les problèmes et les dilemmes déontologiques et éthiques me paraît, par son style, nettement préférable à un code de discipline. Entre autres avantages, elle permet d'échapper aux effets pervers de la maxime expressio unius est exclusio alterius, laquelle exprime une attitude devant les textes très répandue chez les juristes. Elle fournit un document considérablement plus riche par l'information qu'il contient que ne l'est, par exemple, un texte lapidaire comme le Code de déontologie de la magistrature en vigueur au Québec. ${ }^{39}$ Dans sa présentation actuelle, qui est le

36. Ibid. à la p. 316.

37. Supra note 1.

38. Le juge Gonthier a très bien expliqué cette caractéristique de la déontologie dans l'arrêt Ruffo, supra note 22, para. 110, lorsqu'il a écrit : «La règle déontologique [...] se veut une ouverture vers la perfection. Elle est un appel à mieux faire, non par la sujétion à des sanctions diverses mais par l'observation de contraintes personnellement imposées. Une définition, par ailleurs, en déterminant des règles fixes, tend par là même à devenir un plafond, une autorisation implicite de poser les gestes qui ne se veulent pas prohibés. Ces deux notions, sans nul doute, s'avèrent difficiles à réconcilier. Voilà qui explique la généralité du devoir de réserve qui, en tant que norme déontologique, cherche davantage à prodiguer des conseils d'ensemble quant à la conduite que d'en illustrer le détail et les manifestations permises.»

39. Supra note 23. En revanche, cette autre façon d'aborder la déontologie semble avoir été motivée en partie par le souci légitime de ne pas trop peser sur le libre arbitre du juge et sur son indépendance. Dans cette optique, il revient au juge d'interpréter les injonctions générales du Code selon sa conscience, et on ne lui imposera pas les directives précises que l'on retrouve, par exemple, dans un code de déontologie policière (comme le Règlement sur 
fruit de travaux prolongés, ce texte devrait donc alerter les intéressés sur les situations à risque, puis susciter et guider la discussion en vue d'une solution. Enfin, élément essentiel de l'ensemble, l'adoption des Principes coïncide avec la création d'un comité consultatif de la magistrature qui sera appelé à donner des avis sur des cas particuliers. La déontologie est effectivement une casuistique, ${ }^{40}$ mot que j'emploie ici dans un sens dénué de toute nuance péjorative; il faut donc laisser place à la discussion entre gens informés, car il n'est pas réaliste de vouloir traiter d'éthique sur le ton à la fois tatillon et tranchant qu'adopte le législateur dans les lois fiscales. Bref, la seule modeste critique que j'exprimerais sur la forme des Principes concerne leur version française. $^{41}$

2. Sur le fond, les Principes traitent longuement, comme c'était à prévoir dans un texte de ce genre destiné à des juges, de la question de l'impartialité et des conflits d'intérêt. Il n'est pas inutile de noter au passage que, guidé par ce document, Lord Hoffman n'aurait pas connu les déboires que l'on sait dans

la déontologie et la discipline des policiers de la Communauté urbaine de Montréal, R.R.Q. c. C-37.2r1). On ne peut nier que cet argument ait du poids. Il me semble pourtant que, lorsque le code en question s'insère dans un cadre disciplinaire, comme c'est le cas du Code de déontologie de la magistrature en vigueur au Québec, un style moins lapidaire présenterait l'avantage de régler par anticipation des débats peut-être inutiles; un code moins concis aurait peut-être permis d'éviter l'affaire Ruffo, supra note 22.

40. André Lalande, dans son Vocabulaire technique et critique de la philosophie, 14e éd., Paris, Presses Universitaires de France, 1983, en donne la définition suivante : «Étude des cas de conscience, c'est-à-dire des problèmes de détail qui résultent de l'application des règles éthiques à chaque circonstances particulière. [...] Les casuistes ayant été, en général, des théologiens, le mot s'applique surtout à l'éthique dans ses rapports avec la religion. Il se prend souvent d'un sens péjoratif, parce qu'on reproche aux casuistes d'aboutir, par des subtilités de logique, à justifier n'importe quels actes.»

41. Le document donne l'impression générale d'avoir été publié en anglais et en traduit. Je définirais le traduit comme cette langue que l'on utilise beaucoup au Canada et qui consiste en du français ou de l'anglais, dont on a systématiquement expurgé tout indice du génie de la langue. D'emblée, traduire ethics par déontologie n'est pas entièrement satisfaisant, surtout lorsque le document a les finalités qu'on lui prête ici. Quelques mots d'explication auraient été appropriés, bien qu'utiles seulement en français, ce qui aurait contrevenu au triste dogme de la parfaite égalité des textes. Mais cette critique ne se veut pas le moindrement malveillante. Au rayon des documents officiels en traduit, on a vu cent fois pire - par exemple, le Code civil du Québec dans la version entrée en vigueur en 1994. Voir à ce sujet $\mathrm{P}$. Legrand, «Codification and the politics of exclusion : a challenge for comparativists» (1998), 31 U. C. Davis L. Rev. 799, et notamment la note 12 à la p. 803. 
l'affaire Pinochet. ${ }^{42}$ Très actuel par son contenu, le document évoque, bien que ce soit parfois à mots couverts, des situations récentes et controversées qui ont pu nuire à la perception de la magistrature au sein du public. Ainsi, il met en garde contre la banalisation ${ }^{43}$ «du principe de l'indépendance de la magistrature en l'invoquant abusivement, et systématiquement, pour s'opposer aux propositions de changement visant leur structures institutionnelles.» ${ }^{44}$ Il exprime aussi des réserves sur la participation des juges à des commissions d'enquête, à moins qu'ils aient «soigneusement examin[é] leur mandat, ainsi que les facteurs en cause, tels que le temps et les ressources dont ils disposent.» ${ }^{45}$

Cela dit, en accord avec l'air du temps, le document se distingue des autres textes comparables (dont au premier chef les deux Codes of Judicial Conduct américains ${ }^{46}$ ) parce qu'il range l'égalité parmi les cinq principes fondamentaux de la déontologie judiciaire. Cette innovation coïncide avec les préoccupations politiques ${ }^{47}$ de l'époque actuelle, mais l'on sent par endroits,

42. Voir Re Pinochet Ugarte, [1998] H.L.J. No. 41 et Re Pinochet Ugarte, [1998] H.L.J. No. 52. Le principe 6 (Impartialité) C.1.d) énonce : «Les juges évitent toute participation à des causes ou à des organisations susceptibles d'être impliquées dans un litige.»

43. Trivializing, dit l'anglais, ce qui paraît plus près de l'idée d'origine : on invoque le principe d'une manière qui le tourne en dérision.

44. On pense ici au différend entre le ministère de la Justice et les juges du Québec au sujet des tarifs de stationnement auxquels seraient assujettis ces derniers : voir Bisson c. Québec ( $P$. G.), [1992] R.J.Q. 1947 (C.S.) et [1993] R.J.Q. 2581 (C.S.), ainsi que les déclarations du juge Michaud, alors juge en chef adjoint de la Cour supérieure, à la journaliste Rollande Parent : «Se défendant de parler pour l'ensemble des juges, M. Michaud est toutefois prêt à reconnaître que la bataille menée par les juges pour éviter une hausse substantielle de leurs frais de stationnement, sous le couvert de l'indépendance judiciaire, a été une erreur stratégique. "Je crois qu'on a peut-être un peu banalisé un concept aussi important comme celui de l'indépendance judiciaire.”» Presse Canadienne, le 24 août 1994.

45. On pense ici à la controverse autour de la Commission d'enquête sur le déploiement des Forces canadiennes en Somalie : voir Dixon cv. Canada (Commission of Inquiry into the Deployment of Canadian Forces to Somalia) (1997), 146 D.L.R. (4') 156 (C.F.) et (1997), 149 D.L.R. (4 $\left.4^{\mathrm{e}}\right) 269$ (C.F.A.).

46. Le American Bar Association Model Code of Judicial Conduct de 1990 et le American Bar Association Code of Judicial Conduct de 1972. Dans les deux cas, le mot equality n'apparaît nulle part; dans les deux cas, la notion de discrimination n'est abordée que sous l'angle de l'appartenance à des associations ou regroupements pratiquant des formes répréhensibles d'exclusion.

47. Au sens de public policy. 
notamment dans les commentaires sur la formation des juges ${ }^{48}$ et sur leur obligation de se dissocier de certains comportements adoptés en leur présence, ${ }^{49}$ les tensions qui résultent de cette inclusion. Une réflexion fondamentale doit d'ailleurs se poursuivre sur la possibilité qu'il y ait de loin en loin une incompatibilité entre, d'une part, le fait de juger et, d'autre part, les postulats, de plus en plus forts de nos jours dans l'espace public canadien, d'une idéologie égalitaire. ${ }^{50}$ Contraint de ne jamais remettre en question ces postulats, le juge risquerait à l'occasion de ne pas pouvoir exercer librement le discernement qu'on attend de sa part, et qui est garant de l'idée de justice. ${ }^{51}$ Des incidents

48. Supra note 37 à la p. 25 : «Compte tenu des exigences de l'indépendance et de l'impartialité, ce ne sont pas nécessairement tous les types de formation ou outils de formation qui pourront être employés à cette fin. Les juges ne doivent cependant pas être paralysés par de tels dangers. Il faut éviter que des préoccupations d'image exagérées ou non fondées ne minent les efforts déployés pour parfaire la formation des juges.»

49. Ibid.

50. Il est assez naturel qu'un jugement explicite et réprobateur sur la crédibilité d'une partie ou d'un témoin soit ressenti comme blessant par la personne visée, à moins que celle-ci ne considère elle-même que la critique est méritée. Mais la soumission devant la critique n'est pas la règle. Or, on s'attend de la part du juge à des jugements discriminants (entendu au sens didactique) : voir, parmi des quantités d'exemples, $R$. v. Kelly, [1999] O.J. no 1781 , notamment au para. 183. Ne s'expose-t-on pas alors à l'accusation d'avoir «manqué de sensibilité» (showing insensitivity, selon l'expression anglaise, supra note 17, commentaire 4 sous le principe 5)? Voir aussi, qui illustrent cette tension entre l'exercice de la faculté de juger et la perception par le justiciable d'avoir été traité sans préjugé, Mahendran c. Canada (Minister of Employment \& Immigration) (1991), 14 Imm. L.R. (2d) 30 (C.A.F), Peraza v. Canada (Minister of Citizenship and Immigration), [1994] F.C.J. $\mathrm{n}^{\circ} 1551$ (C.F.), Saleh v. Canada (Minister of Citizenship and Immigration), [1995] F.C.J. $n^{\circ} 745$ (C.F.) et Villalobos v. Canada (Minister of Citizenship and Immigration), [1999] F.C.J. $\mathrm{n}^{\circ} 806$ (C.F.).

51. Jusqu'ici, les juges canadiens semblent bien avoir résisté sans trop de difficulté à ce que certains dénoncent comme les excès de la «rectitude politique». Bien au contraire, lorsqu'on les a pris en défaut, comme ce fut le cas tout récemment du juge John McClung (voir le communiqué du Conseil canadien de la magistrature en date du 21 mai 1999), c'était pour les excès inverses. Mais la question se posera tôt ou tard d'une manière qui fera ressortir les visées de l'idéologie égalitaire et les conséquences normatives qui en découlent. Plusieurs arrêts récents, et pas seulement sur le droit à l'égalité, en donnent des signes avant-coureurs. Voir par exemple $R$. c. Gladue, [1999] 1 R.C.S. 688, [1999] A.C.S. $n^{\circ} 19$, en ligne : QL (A.C.S.), en particulier aux para. 61-64 : la nette surreprésentation des autochtones dans les systèmes pénitentiaires fédéral et provinciaux du Canada est attribuée à une discrimination systémique issue de préjugés contre les autochtones. La Cour cite à ce sujet le rapport d'une commission royale d'enquête : «Ce lamentable échec découle surtout de ce qu'autochtones et non-autochtones affichent des conceptions extrêmement différentes à l'égard de questions fondamentales comme la nature de la justice et la façon de l'administrer.» Selon ce 
très divers, comme ceux qui ont mis en cause la juge Moreau-Bérubé de la Cour provinciale du Nouveau-Brunswick en $1999,{ }^{52}$ le juge Francis Muldoon de la Cour fédérale en $1998^{53}$ ou la juge Corinne Sparks du Tribunal de la jeunesse de Nouvelle-Écosse en $1994,{ }^{54}$ montrent combien le fait de porter un jugement risque de heurter celui ou celle qui en est la cible, et combien il importe pour le juge de ne jamais paraître sous l'influence d'un préjugé quelconque. Cela ne signifie pas pour autant que toute distance prise par rapport au principe d'égalité

raisonnement, le traitement statistiquement inégalitaire des autochtones dans le système carcéral est le fruit d'une discrimination en l'absence de laquelle ce traitement, présume-ton, serait statistiquement égalitaire, c'est à dire que le taux d'incarcération serait proportionnel au poids démographique relatif des autochtones dans la population canadienne. L'analyse est séduisante mais vide-t-elle la question? Celui qui voudrait aller au-delà de cette analyse risquerait-il de «manquer de sensibilité», selon la litote des Principes de déontologie judiciaire déjà citée plus haut?

52. Voir la dépêche de la Presse Canadienne en date du 15 avril 1999, «Controversial judge removed from bench», ainsi que la décision du Conseil de la magistrature du NouveauBrunswick en date du 9 avril 1999. Le Conseil cite comme suit les propos de la juge Moreau-Bérubé, prononcés lors d'un jugement sur sentence : «If a survey were taken in the Acadian Peninsula, of the honest people as against the dishonest people, I have the impression that the dishonest people would win. We have now got to the point where we can no longer trust our neighbour next door or across the street. In the area where I live, I wonder whether I'm not myself surrounded by crooks. And, that is how people live in the Peninsula, but we point the finger at outsiders. Ah, we don't like to be singled out in the Peninsula. And it makes me sad to say this because I live in the Peninsula now. It's my home. But look at the honest people in the Peninsula, they are very few and far between, and they are becoming fewer and fewer.» Malgré des excuses publiques et apparemment sincères, la juge Moreau-Bérubé fut démise de ses fonctions, faisant ainsi mentir l'adage «faute avouée est à demi pardonnée». Ces propos étaient sûrement intempestifs, mais ne confond-on pas ici une défaillance ou un écart de conduite, même sérieux, avec la faute grave justifiant la destitution? N'y a-t-il pas danger que, tenus à peine de destitution de ne jamais commettre de telles erreurs, les juges s'abstiennent de porter des jugements impopulaires, même justifiés, de crainte de susciter une réaction trop hostile? Voir et comparer Anctil c. Pearson, [1974] C.A. 19, pour une autre approche, peut-être plus souhaitable, à ce genre de problème.

53. Décision d'un comité d'enquête du Conseil canadien de la magistrature en date du 11 mars 1998. Le comité écrit (p. 3) : «Your use of such needlessly inflammatory, derogatory and culturally insensitive language on matters of little or no relevance to the determination of the case leaves the impression that you used your judicial office inappropriately to express your personal opinions in relation to aboriginal rights.» Comparer avec $R$. v. Brertton, [1998] 8 W.W.R. 170 (Alta Q.B.); il y a, c'est incontestable, une nette différence de degré entre ces deux espèces.

54. Voir R. c. S.(R.D.), [1997] 3 R.C.S. 484. Les circonstances de cette affaire la distinguent des deux précédentes, car il allait de soi ici qu'une plainte déontologique ou disciplinaire contre le juge n'aurait pas de raison d'être. 
constitue un préjugé anti-égalitaire et que tout jugement critique à l'endroit de quelqu'un, ou même d'un groupe de personnes, brime un droit à l'égalité. Sans doute faudra-t-il laisser le temps faire son oeuvre. Cela se produit déjà au fil des décisions. Quelques-unes, parce que c'est désormais nécessaire, ont commencé à circonscrire un espace où le juge peut s'exprimer sans ambages, et cela même si les propos qu'il tient indisposent certains et les incitent à mettre en doute son impartialité. ${ }^{55}$

Concluons cette partie de l'exposé avec deux ou trois observations additionnelles sur ce que la dynamique de l'égalité implique pour le juge. L'incident McClung a fait couler beaucoup d'encre en 1999 et s'est soldé par un blâme porté par un sous-comité du Conseil canadien de la magistrature contre l'intéressé, juge de la Cour d'appel de l'Alberta. ${ }^{56}$ Entre autres choses dignes d'intérêt, ce dossier recèle un élément qui met en relief l'évolution des sociétés libérales à la fin du $\mathrm{XX}^{\mathrm{e}}$ siècle et la difficile transition quelle impose dans le contenu du discours judiciaire. Pas moins de 24 plaintes avaient été déposées contre le juge McClung en raison d'une lettre ouverte qu'il avait publiée dans le quotidien The National Post ainsi que d'une entrevue accordée peu de temps après au même journal. Néanmoins, toutes ces plaintes ne visaient pas la lettre et l'entrevue en question, indiscrétions extrajudiciaires. L'une d'entre elles concernait la conduite judiciaire du juge McClung et lui faisait grief de certains

55. Voir les communiqués du Conseil canadien de la magistrature en date du 1er avril 1999 (répondant à une plainte de l'association Real Women of Canada contre le juge L'HeureuxDubé) et en date du 23 janvier 1998 (répondant à une plainte du Chinese Canadian National Council contre le juge en chef Lamer). Dans ce second cas, qui faisait suite à un échange de questions pendant l'audition du pourvoi dans l'affaire S.(R.D.), ibid., l'incident avait suscité des commentaires d'une partie à l'instance : voir la dépêche de la Presse Canadienne en date du 4 novembre 1997 : «Yolah Grant, a lawyer for the National Organization of Immigrant and Visible Minority Women and for Legal Education and Action Fund, was at the appeal hearing. "I thought it was inappropriate and unbecoming for that office for [Chief Justice Lamer] to make those comments in a seemingly offhand manner," said Grant. Lamer apologized to the Canadian Chinese National Council in a letter faxed just minutes before the group's announcement, said lawyer Avvy Go, director of the Metro Toronto Chinese and South East Asian Legal Clinic. But Go said the apology alone is not enough. The National Action Committee on the Status of Women has called on Lamer to step down.»

56. «Le sous-comité exprime sa vive réprobation au sujet de la conduite du juge McClung» (21 mai 1999), 98-128, en ligne : Conseil canadien de la magistrature <http://www.cjcccm.gc.ca/francais/communiques.htm> (date d'accès : 27 novembre 1999. 
passages dans le jugement Vriend.$^{57}$ Le juge McClung y écrivait que l'inclusion de l'orientation sexuelle dans la législation albertaine sur les droits de la personne ferait de la sodomie un droit fondamental et que ce résultat irait à l'encontre d'un millénaire de magistère moral. ${ }^{58}$ Plus loin dans les mêmes motifs, il ajoutait une allusion à des criminels notoires (Dahmer, Bernardo, Olson) dans le but de signaler, semble-t-il, l'hostilité probable du public et des membres de la législature envers toute mesure de libéralisation en faveur de l'orientation sexuelle. ${ }^{59}$ Le Conseil, par l'entremise du sous-comité formé à cette fin, condamne ces commentaires. Dans la lettre qu'il adresse au juge McClung, il écrit : «[traduction] ... vos commentaires dépassaient la limite de la grande latitude dont les juges bénéficient lorsqu'ils rédigent leurs motifs. Ils n'ont aucun lien logique avec les questions en litige et vont à l'encontre du respect des droits à l'égalité. Par conséquent, ils constituent une conduite inappropriée de la part d'un juge.» ${ }^{60}$

On a donc ici le spectacle malheureux d'un juge qui a perdu, du moins pour un temps, le contrôle de sa rhétorique. ${ }^{61}$ Faut-il se surprendre du blâme qu'il s'attire? Non. Cette rhétorique est blessante pour une minorité qui a conquis difficilement un droit à la différence et à l'égalité devant la loi. En tenant les propos qu'on lui reproche, le juge McClung ignore et déprécie une évolution commencée il y a plus de trente ans par le retrait d'interdictions pénales d'une sphère de la vie privée, et qui se poursuit aujourd'hui en Cour suprême avec des décisions comme les arrêts Vriend ou M. c. $H^{62}$. Quoique

57. Re Vriend (1996), 132 D.L.R. (4e) 595, infirmé par la Cour suprême du Canada dans Vriend c. Alberta, [1998] 1 R.C.S. 493.

58. Le passage se lit en anglais «rebutting a millenia of moral teaching». Re Vriend, ibid. à la p. 609.

59. «It is pointless to deny that the Dahmer, Bernardo and Clifford Robert Olsen prosecutions have recently heightened public concern about violently aberrant sexual configurations and how they find expression against their victims.»Ibid. à la p. 611.

60. Supra note 56 et voir la lettre en date du 19 mai 1999.

61. Ç'aurait pu être plus grave. Hypothétiquement, les propos du juge McClung auraient pu dénoter de sa part un préjugé contre l'homosexualité. Le comité écarte cette hypothèse, tout en ajoutant : «... ces commentaires étaient inutiles, malheureux et irrévérencieux, mais ils ne démontrent pas une homophobie sous-jacente de votre part.»Ibid., p. 10.

62. Vriend c. Alberta, supra note 57 et M. c. H., [1999] 171 D.L.R. (4') 577, [1999] A.C.S. $\mathrm{n}^{\circ}$ 23, en ligne : QL (A.C.S.). 
cette rhétorique trouve écho dans une jurisprudence moins récente, ${ }^{63}$ la transformation du discours judiciaire est aussi l'un des enjeux de l'évolution vers une société exempte de préjugés hostiles envers les minorités sexuelles. Les signes de cette évolution sont aujourd' hui nombreux. Ainsi, on a vu récemment en Australie l'un des sept membres de la High Court faire discrètement connaître son homosexualité au moyen d'une notice biographique dans le Who's Who ${ }^{64}$ Cette information, qui naguère n' aurait pas été rendue publique, ou qui, l'étant devenue, aurait suscité une controverse sinon un scandale, n'a provoqué en l'occurrence aucune réaction significative. Dans ce contexte, qui est désormais commun à beaucoup de sociétés libérales, des propos courroucés sur la sodomie ou sur les déviations sexuelles au cours d'un débat juridique sur l'orientation sexuelle paraissent axiologiquement anachroniques ${ }^{65}$ et peut-être même malveillants. Qui plus est, un telle rhétorique fait écran à la question de principe pourtant très importante que recelait l'affaire Vriend, soit celle de savoir si le législateur par son inaction peut contrevenir à la constitution.

Mais tous les cas ne sont pas aussi clairs. En tout état de cause, le juge qui s'apprête à rejeter une revendication d'un groupe identitaire défavorisé (y compris la revendication du statut de groupe identitaire défavorisé) doit faire preuve d'une grande circonspection. L'horizon actuel du contentieux sur

63. Le passage sur «a millenia of moral teaching» est tiré des motifs du juge en chef Burger dans le jugement de la Cour suprême des États-Unis, Bowers cv. Hardwick, 92 L. Ed. 2d 140 (1986) à la p. 150. Voir aussi, en droit anglais, Lord Sumner dans Thompson c. R., [191819] All E.R. 521 à la p. 528, et Lord Hailsham of St Marylebone dans D.P.P. c. Boardman, [1974] 3 All. E.R. 887, citant Lord Reid dans D.P.P. c. Kilbourne, [1973] 1 All E.R. 440, au sujet du passage tiré du jugement de Lord Sumner.

64. Voir «Outing of Kirby Welcomed», The Canberra Times, 20 avril 1999, p. A5, et «A NonSecret is Out», The Canberra Times, 22 avril 1999 à la p. A8, mais voir aussi P. Akerman, «Irate Howls Don't Answer the Question», The Daily Telegraph, 8 juin 1999 à la p. 11.

65. Expression malheureusement un peu pédante, mais qui renvoie à quelque chose de spécifique : je pense ici aux irréversibilités dans la sensibilité morale (et dans la rationalité axiologique), idée éloquemment développée dans R. Boudon, «Critique de la bienveillance universelle, ou De la rationalité axiologique», dans G. LaForest et P. de Lara, dir., Charles Taylor et l'interprétation de l'identité moderne, Québec, Presses de l'Université Laval, 1998 à la p. 285 et s. La jubilation de Mme de Sévigné devant la torture, que relatait Tocqueville (ibid. aux pp. 313 et 315), ou encore le propos peut-être apocryphe attribué à Saul Below («Lorsque les Zoulous produiront un Tolstoï, nous le lirons!») dans C. Taylor, Multiculturalisme - Différence et démocratie, trad. par D.-A. Canal, Paris, Flammarion, 1992 aux pp. 62 et 97, sont eux aussi axiologiquement anachroniques. 
l'égalité ne permet pas d'entrevoir aisément le moment où ces litiges cesseront d'être chargés d'émotivité. Entre-temps, sous peine d'être soupçonné de racisme, de sexisme, d'homophobie ou d'un quelconque autre «manque de sensibilité», il faut considérer que tous les attributs d'une identité collective minoritaire ou défavorisée sont positifs (de sorte que, par exemple, dans un contexte officiel, on ne peut parler des Québécois qu'en bien, jamais en mal, car prétendre le contraire, c'est être anti-québécois ${ }^{66}$ ); en tout cas faut-il taire ce qui risquerait d'être perçu comme négatif. Cette attitude, difficilement défendable dans l'abstrait, est rendue nécessaire par la mentalité de l'époque. La tâche du juge devient donc particulièrement délicate dès que sa décision touche de près ou de loin à une question identitaire.

66. Cette question est beaucoup trop complexe pour être traitée convenablement ici. Néanmoins, ajoutons quelques nuances. Le Québécois, comme bien d'autres, peut sûrement jeter sur luimême et sur certains travers de sa culture un regard à la fois critique et amusé : la très grande popularité de productions comme Elvis Gratton ou La petite vie le démontre. Mais tout dépend de qui juge qui. On ne peut nier, par ailleurs, que l'idéologie égalitaire (et le multiculturalisme) traînent dans leur sillage une forme de démission normative. C'est cet obstacle au jugement que vise le philosophe Charles Taylor lorsqu'il écrit : «Il est juste de réclamer comme un droit que l'on aborde l'étude de certaines cultures avec une présomption de leur valeur [...]. Mais il est dépourvu de sens d'exiger comme un droit que nous finissions par conclure que leur valeur est grande ou égale à celle des autres. [... D]es théories subjectivistes - à demi néo-nitzschéennes — sont très souvent invoquées dans ce débat. Dérivant fréquemment de Foucault et de Derrida, elles proclament que tous les jugements de valeur sont fondés sur des critères qui sont, en dernier ressort, imposés (puis renforcés) par des structures de pouvoir. Ce pourquoi ces théories prolifèrent ici devrait être clair. Un jugement favorable sur demande est une ineptie, à moins que certaines de ces théories ne soient recevables. En outre, la délivrance d'un tel jugement sur demande est un acte de condescendance stupéfiant. Personne ne peut réellement y voir un authentique acte de respect. Cela ressemble plus à un acte de respect prétendu, accordé sur l'insistance de son bénéficiaire supposé. Objectivement, un tel acte implique le mépris pour l'intelligence de ce dernier : être l'objet d'un tel acte de respect dévalorise! Les tenants des théories néonietzschéennes espèrent échapper à ce noeud d'hypocrisie en transformant la totalité du problème en une question de pouvoir et de contre-pouvoir. La question n'est donc plus une affaire de respect, mais de parti-pris et de solidarité. Pourtant, c'est difficilement une solution satisfaisante, parce que, en prenant parti pris, on manque la force agissante de ce genre de politique, qui est précisément la recherche de reconnaissance et de respect.» Ibid. aux pp. 93 et 95 . Il y a tellement eu, au XX $\mathrm{XX}^{\mathrm{e}}$ siècle, d'injustices révoltantes fondées sur des préjugés raciaux, sexuels, ethniques et autres, que l'époque nous incite à suspendre tout jugement critique à partir de ces catégories : le jugement est de la sorte devenu préjugé. 
Faut-il souhaiter que le rôle du juge soit compris du public pour ce qu'il est véritablement? Poser la question de cette façon dénote un certain manque d'humilité : qui, en effet, aurait l'outrecuidance de prétendre qu'il n'ignore rien de ce qu'est véritablement le rôle du juge? Aussi, toutes les explications qu'on en donne relèvent-elles à des degrés divers de l'approximation. Néanmoins, je considère pour ma part que l'on doit à Kelsen l'explication la plus succincte et la plus vraisemblable de la fonction judiciaire considérée dans sa nature intime. Cette explication, qu'on trouve dans sa Théorie pure du droit ${ }^{67}$ me paraît avoir le grand mérite de n'exprimer que le strict nécessaire, soit ce qui peut être dit avec le maximum de rigueur. Kelsen

67. Je veux parler ici du titre VIII, «L'interprétation», dans H. Kelson, Théorie pure du droit, trad. par C. Eisemann, Paris, Dalloz, 1962 à la p. 453 et s. Kelsen y traite de l'interprétation des normes juridiques, mais, à mon sens, l'«acte de volonté» dont il parle (p. 460), et qui s'unit à l' «opération de connaissance», vaut autant pour les conclusions de fait que pour les conclusions de droit. Peut-être seulement faut-il tempérer cette dernière affirmation en ajoutant que, s'agissant de faits, la règle du fardeau de la preuve se substituera parfois à l'acte de volonté. Mais la distinction droit/fait est poreuse et il existe des situations où, aussi inorthodoxe que cela puisse paraître, la partie en demande a en quelque sorte le fardeau de la preuve sur le droit, ou du moins le fardeau d'induire chez le juge un degré de conviction plus fort que d'habitude : qu'est-ce en effet que l'idée d'une apparence de droit suffisante en matière d'injonction interlocutoire? (Voir sur ce point B. Cliche et D. Ferland, «L'injonction», dans D. Ferland et B. Émery, dir., Précis de procédure civile du Québec, $3^{\mathrm{e}}$ éd., vol. 2, Cowansville (Qc.), Yvon Blais, 1997, 340, à la p. 383 et s.). Dans ces situations, où ce que le droit enseigne n'est pas discernable et ne peut donc se prêter à une pure opération de connaissance, la règle de l'apparence de droit suffisante dispense le juge de l'acte de volonté dont parle Kelsen. En l'absence d'une telle règle, le régime général prévaut et (1) soit que ce que le droit enseigné résoud sans laisser de place au doute la question débattue entre les parties, auquel cas, (a) elles finissent par s'y conformer d'elles-mêmes ou (b) le tribunal, informé de ce que le droit lui dicte (the core), intervient contre la partie récalcitrante en une besogne de police et la contraint à s'y conformer au moyen d'une mesure d'exécution, (2) soit que ce que le droit enseigne ne suffit pas à résoudre cette question, auquel cas le juge, après s'être renseigné sur l'état du droit (the penumbra), fait acte de volonté et résoud la question en complétant la norme interstitially, comme le disait sagacement feu Oliver Wendell Holmes fils. Au fond, le système juridique tient la route grâce aux rustines du droit prétorien. On trouvera une explication plus récente de la place de l'interprétation en droit, explication qui me paraît largement fondée, dans D. Patterson, Law and Truth, New York et Oxford, Oxford University Press, 1996, en particulier au chapitre 5, «Law as Interpretation : The Jurisprudence of Ronald Dworkin». Le lecteur désireux de se divertir pourra lire avec égal bonheur le chapitre 6, «Law as Interpretive Community : The Case of Stanley Fish», mais la thèse de Fish, moins nuancée que celle de Dworkin, me paraît moins susceptible de rallier la magistrature. 
et Hart se rejoignent d'ailleurs sur ce point essentiel. ${ }^{68}$ Il y a eu au $\mathrm{XX}^{\mathrm{e}}$ siècle un foisonnement de théories politiques et philosophiques sur la justice, mais beaucoup moins de choses se sont écrites sur l'exercice par le juge de la faculté de juger, ${ }^{69}$ surtout lorsqu' on laisse de côté le débat nourri mais parfois oiseux en droit américain sur la question spécifique de la légitimité du juge

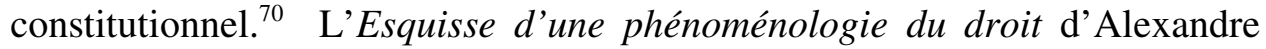
Kojève $^{71}$ est un de ces essais qui mettent le juge sous la lunette du microscope, non pas tant parce que cet auteur s'intéresse au juge en tant que tel, mais parce que sa définition du droit place le juge impartial et désintéressé au coeur du droit, en fait le révélateur même de la juridicité. Le droit étant «l'application d'une certaine idée de justice à des interactions sociales données ${ }^{72}$ et la spécifité du Droit résid[ant] précisément dans la présence de ce tiers», ${ }^{73}$ son contenu n'a pas d'importance, ce n'est pas ce qui lui confère sa juridicité. Aussi, la définition du droit «ne donne[-t-elle] aucun renseignement sur le contenu du Droit, quel qu'il soit. Elle permet seulement de voir si un contenu donné (un phénomène) est ou non authentiquement juridique. Et elle permet de le faire quel que soit ce contenu. C'est pourquoi on peut dire que c'est une définition

68. Hart pousse plus loin l'étude de la fonction judiciaire, et le fait dans un mode angloaméricain, en ce sens qu'il répond aux «réalistes» américains (rule-sceptics) avec l'allégorie célèbre du marqueur (scorer) : voir H.L.A. Hart, supra note 2 à la p. 141 et s., et en particulier à la p. 145 : «The open texture of law leaves to courts a law creating power far wider and more important than that left to scorers, whose decisions are not used as lawmaking precedents. Whatever courts decide, both on matters lying within that part of the rule which seems plain to all, and those lying on its debattable border, stands till altered by legislation; and over the interpretation of that, courts will again havethe same last authoritative voice. Nonetheless there still remains a distinction between a constitution which, after setting up a system of courts, provides that the law shall be whatever the supreme court thinks fit, and the actual constitution of the United States — or for that matter the constitution of any modern State.»

69. Je pense ici, par exemple, à des descriptions comme celles (provocantes en l'occurrence, pour ne pas dire subversive) de Jerome Frank (le futur juge Jerome Frank) dans «What Courts do in Fact» (1932), 26 U. Ill. L. Rev. 645.

70. Ce débat, qui porte principalement sur la légitimité du jugement judiciaire, se poursuit, et les travaux sur le sujet sont surabondants. Pour une synthèse succincte et mesurée, voir $\mathrm{P}$. W. Kahn, Legitimacy and History - Self-Government in American Constitutional Theory, New Haven, Yale University Press, 1992 à la p. 134 et s.

71. Paris, Gallimard, 1981 (rédigé en 1943).

72. Ibid. à la p. 200.

73. Ibid. à la p. 191. 
formelle (d'ailleurs behavioriste) de l'essence Droit.» ${ }^{74}$ Bien qu'il cherche à circonscrire le droit par son contenu, le juriste et philosophe français Michel Villey accorde lui aussi au processus dialectique de la décision de justice une signification décisive. ${ }^{75}$ Un bon nombre de réflexions du même ordre tendent elles-aussi à démontrer que des conditions d'intégrité, de compétence générale, de connaissance du droit, de régularité procédurale et d'impartialité concourent à asseoir l'autorité du juge, mais que, une fois satisfaites ces conditions étrangères au fond du débat judiciaire, la seule légitimité du juge tient au fait qu'il faut un juge pour mettre fin à certaines controverses. ${ }^{76}$ Sa légitimité ne découle jamais, et ne peut donc en aucun cas dépendre, de la conformité de la décision rendue avec un quelconque idéal platonique de décision «juste» ou «conforme au droit» qui préexisterait pour les fins du litige hic et nunc à la décision qui en dispose. Énoncer ces choses ne signifie évidemment pas que les décisions des tribunaux ne sont pas justes : puisque tout est mis en oeuvre pour qu'elles le soient, il faut conclure qu'elles le sont la plupart du temps, mais il n'y a aucun moyen de démontrer avec certitude que c'est effectivement le cas. En somme, là n'est pas la question pour le juriste.

74. Ibid. à la p. 93 [les italiques sont dans l'original].

75. Villey n'a jamais caché son hostilité envers le positivisme kelsénien (sur cette notion, voir H.L.A. Hart, supra note 2 à la p. 253, note sur la page 181), mais il professe par ailleurs une conception de la décision de justice qui n'est pas incompatible avec celle de Kelsen : simplement, Kelsen abandonne à la seule volonté du juge ce qui pour Villey doit procéder d'une recherche dialectique du juste conformément au droit naturel. («Tel qu'il vient d'être défini, le droit naturel a le défaut d'être dans les choses, ou plutôt existe dans les choses une tendance à le réaliser. Il n'a pas été démontré encore qu'il fût présent dans nos consciences.» Michel Villey, Questions de Saint Thomas sur le droit et la politique, Paris, Presses Universitaires de France, 1987 à la p. 152 (les italiques sont dans l'original) et, plus généralement à la p.145 et $\mathrm{s}$. : «Kelsen réfuté».)

76. Reconnaissons que cet énoncé n'est pas simple. Je veux dire par là que la légitimité du juge dépend en tout et pour tout de son comportement scrupuleux de juge, après avoir été régulièrement désigné pour se décharger de cette responsabilité par une autorité compétente qui l'a nommé pour son impartialité. Dès lors, sa décision, étant donné la fréquente, et certains iraient jusqu'à dire la constante indétermination de ce qu'on lui soumet, peut tomber dans un sens ou dans l'autre. Mais cela n'a pas d'importance, car les conditions de sa légitimité sont déjà remplies. Juger, en somme, tient inévitablement de la pétition de principe. 
Les travaux de science politique ou de sociologie des organisations, comme le récent et fort intéressant Final Appeal ${ }^{77}$ de cinq politologues canadiens, nous apprennent beaucoup de choses sur la gestion interne des tribunaux, sur les habitudes de travail des juges et sur une foule d'autres sujets connexes, mais, s'agissant du rôle du juge dans son principe essentiel, ces travaux nous renseignent seulement sur ce que les juges pensent de ce qu'ils font. ${ }^{78}$ La question, d'ailleurs, ne fait pas l'unanimité parmi eux.

En fin de compte, hors les besognes de police, ${ }^{79}$ les décisions judiciaires sur le droit sont de l'ordre du volitif plutôt que du cognitif : les juges agissent comme des législateurs, mais à la pièce, petit à petit, afin de combler les innombrables lacunes du droit et compléter plutôt qu' «interpréter» le sens de textes qui ne disent jamais tout. Certes, la connaissance du droit élimine diverses

77. I. Greene et al., Final Appeal - Decision-Making in Canadian Courts of Appeal, Toronto, James Lorimer \& Company, 1998.

78. Ibid. aux pp. 181-198, et notamment, à la p. 187 : «We asked the judges whether they thought the Charter had created a "crisis of legitimacy" for the courts - a phrase which was used by some judges in the mid-1980s to describe their fears about judges becoming involved in lawmaking through high-profile Charter of Rights cases. The judges were evenly split on this issue, with thirty-three of seventy thinking that such a crisis existed (45 per cent), and thirty-two saying that there was no crisis; the remainder were neutral. The twentytwo judges who described themselves fundamentally as law interpreters were more likely to think that there was a crisis of legitimacy than those who perceived themselves as having a mixed lawmaking and law-interpreting role.» Si le débat porte sur l'état véritable des choses, que conclure de ces réponses?

79. Voir supra note 67 in fine. Est-il besoin de préciser que l'expression est employée ici dans un sens métaphorique. Il est difficile de concevoir une décision de la Cour suprême du Canada qui correspondrait à cette description (sauf peut-être certains jugements rejetant une requête pour permission d'appeler dans les cas où un justiciable s'obstine à faire valoir des arguments insanes). La même observation vaudra dans la quasi-totalité des cas pour les décisions des cours d'appel de premier niveau. En première instance, les choses sont plus nuancées. Beaucoup de procès soulèvent des questions de droit qui ne sont pas simples. En matière pénale, par exemple, certains procès devant jury recèlent des quantités de difficultés qui mettent lourdement à contribution le savoir et la créativité (donc la volonté) du juge. Mais il reste que, bon en mal, un énorme corpus d'affaires ex parte, par défaut, sur plaidoyer de culpabilité, voire même contestées, comporte pour l'essentiel l'application de normes claires à des faits non-litigieux; le juge est alors une figure d'autorité, il voit à la sanction de la loi, sans plus. 
hypothèses du champ des décisions possibles ${ }^{80}$ et elle conduit les juges au point où certains choix seulement s'offrent à eux, des choix tous potentiellement conformes au droit, mais contradictoires également puisque proposés par des parties opposées. Celui qu'ils retiennent, parfois unanimement, ${ }^{81}$ mais assez souvent aussi par une mince majorité, devient ipso facto le seul choix conforme au droit. Il prévaudra dorénavant sur tous les autres et le débat judiciaire entamé en amont de ce choix pourra maintenant se poursuivre si besoin est en aval et sur autre chose. Cela n'exclut aucunement que les juges choisissent ce qui leur paraît juste «en leur âme et conscience», périphrase surannée, mais expressive. Voici donc la seule chose à laquelle le justiciable en procès a toujours droit : la totale intégrité du juge. Les juges ont intérêt, aussi, à exercer leur autorité judicieusement, avec retenue, modération et circonspection, car leur légitimité, opératoire plutôt que substantielle, est fragile. Personne, par contre, n'a encore découvert le déterminisme secret qui dicterait leurs choix. On continuera dans l'université à réfléchir sur cette question bien que la réponse somme toute assez modeste qu'y apporte Kelsen paraisse, sinon indépassable, du moins à la limite actuelle du dicible sur ce problème. ${ }^{82}$ Attendons de voir si la neuroscience nous apprendra bientôt quelque chose de neuf à ce sujet. J'en doute.

Que cette problématique fondamentale conserve aujourd'hui toute son actualité, même dans les replis les plus austères du droit positif, est incontestable. C'est ce que démontre de façon frappante le récent arrêt de la Chambre des Lords dans l'affaire Kleinwort Benson Ltd. c. Lincoln City

80. C'est le problème assez difficile de la prétention de droit manifestement mal fondée, prétention qui peut avoir son origine dans une pathologie souvent observée par les tribunaux, la quérulence.

81. ... et la dissidence n'existe pas pour le juge de première instance siégeant seul.

82. Tant de choses lucides s'écrivent sur le sujet qu'on a l'embarras du choix. Voir entre autres, pour la qualité du débat, A. T. Arulanantham, «Breaking the Rules : Wittgenstein and Legal Realism» (1998), 107 Yale L.J. 1853, A. C. Hutchinson, «Going Places — Moving Beyond Crisis» (1999), 49 U. Toronto L.J. 249 (pour une critique vigoureuse de M. Rosenfeld, Just Interpretations; Law between Ethics and Politics, Berkeley, University of California Press, 1998) et, pour qui n'a pas froid aux yeux, P. Nonet, «Judgment» (1995), 48 Van. L. R. 987, qui entame sa réflexion avec le constat (p. 988) «There are, and there can be, no rules regarding the application of rules. If Kant is right, a sizable part of what we take to be "law," and almost all jurisprudence, are nothing but a futile striving to overcome this essential unruliness of judgment.» La démonstration qui suit l'affirmation est ici encore plutôt convaincante. 
Council..$^{83}$ Très ciselée, selon la forme que pratique cette juridiction, la décision rendue à la majorité de trois des cinq Law Lords a le grand mérite d'aborder les choses de front et de trancher la question avec franchise. On peut énoncer en deux propositions simples ce qu'elle enseigne sur ce qui nous intéresse ici ${ }^{84}$ : (1) une décision de justice a bel et bien un effet rétroactif car, même si elle est tenue pour énoncer le droit comme il existait au moment où le litige a pris forme, il s'agit là d'une fiction; (2) le paiement effectué de bonne foi par une partie qui croit y être tenue en vertu du droit alors en vigueur sera considéré avoir été effectué par erreur si une décision de justice entre des parties différentes vient par la suite fixer le droit en sens contraire. La première proposition fait l'unanimité, la seconde recueille l'adhésion de la majorité. On voit donc ici la réalité rejoindre la fiction, et ce de deux façons distinctes : elle s'impose à l'entendement d'une manière qui force le rejet d'une fiction séculaire sur la nature déclaratoire du droit prétorien ${ }^{85}$ et, de manière peut-être plus malicieuse, elle donne raison à Sir Alan Herbert d'avoir posé dans une oeuvre de fiction la question plutôt embarrassante : «Is a decision of the House of Lords an Act of God?» ${ }^{86}$ Une question litigieuse contraint le tribunal à départager des

83. [1998] H.L.J. No. 39.

84. L'arrêt dispose aussi au fond d'une importante question de droit privé et il établit que désormais, dans une action en restitution (ou, selon la terminologie civiliste, une action pour réception de l'indu), l'erreur de droit du demandeur ne constituera plus une cause d'exonération pour le défendeur.

85. Comme le dit Lord Goff of Chieveley, para. $65:$ «... we all know that in reality, in the common law as in equity, the law is the subject of development by the judges - normally, ofcourse, by appellate judges. [...] The recognition that this is what actually happens requires, however, that we should look at the declaratory theory of judicial decision with open eyes and reinterpret it in the light of the way in which all judges, common law and equity, actually decide cases today.» et para. 69 «The historical theory of judicial decision, though it may in the past have served its purpose, was indeed a fiction. But it does mean that, when the judges state what the law is, their decisions do, in the sense I have described, have a retrospective effect.» Supra note 83.

86. V Voir Dahlia, Ltd. C. Yvonne, dans Uncommon law; being sixty-six misleading cases, $7^{\mathrm{e}}$ éd., Londres, Methuen \& Co., 1950 à la p. 314 (un ouvrage dont la publication fut certainement l'un des hauts moments de l'humour juridique). Cette idée affleure dans le jugement de Lord Hoffman, para. 134 et 135, lorsqu'il écrit : «At the end of argument I was of opinion, perhaps not in a very focused way, that a person who pays in accordance with what was then a settled view of the law has not made a mistake. In fact it seemed to me that one could go further and say that if he had acted in accordance with a tenable view of the law, he had not made a mistake. In the first place he was right and in the second neither right nor wrong, but in both cases his state of mind could be better described as a failure to predict some future 
propositions qui sont pour la plupart problématiques ${ }^{87}$, parfois assertoriques ${ }^{88}$, mais presque jamais apodictiques. ${ }^{89}$ Cela tient à la nature des questions dont un tribunal est susceptible d'être saisi.

Je ne suis pas sûr, pour ma part, qu'il soit utile de dévoiler tous ces secrets de fabrication sur la place publique. Même s'ils étaient dévoilés, je ne suis par sûr qu'ils seraient bien compris de tous. S'ils étaient compris, je ne suis pas sûr que les tribunaux survivraient sans dommage à l'expérience. Comme je suis personnellement convaincu que les tribunaux, sous la forme que j'ai décrite, sont absolument nécessaires pour qu'une société jouisse de l'état de droit, je ne souhaite pas tenter l'expérience. Néanmoins, il est souhaitable que les juges sachent combien est fragile leur pouvoir pour qu'ils en usent sans excès. Quant

event (scilicet, a decision of this House) than a mistake about the existing state of the law. [...] On reflection, however, I have come to the conclusion that this theory was wrong...» La même théorie, plus fataliste sans doute que celle retenue en fin de compte par Lord Hoffman, semble avoir été implicitement adoptée par Lord Browne-Wilkinson dans sa dissidence : voir para. 15.

87. «Nous vivons en démocratie.»

88. «Il existe une planète Terre.» C'est en général le domaine du contentieux sur les faits, à cette importante nuance près que, dans la vérification de ces propositions, la tâche du tribunal est considérablement facilitée par la règle du fardeau de la preuve. Celle-ci permet de trancher la question lorsqu'on ne parvient pas à élucider ce qui s'est véritablement produit. Mais une proposition rejetée parce qu'elle n'est pas prouvée n'est pas nécessairement une proposition fausse. Ainsi s'explique la différence entre les verdicts de Not guilty et Not proven en droit écossais.

89. $« \mathrm{~A}=\mathrm{B}=\mathrm{C}$; donc, $\mathrm{A}=\mathrm{C}$.» La décision de justice peut être constitutive de propositions apodictiques qui étaient problématiques en amont du jugement. Ainsi, ont sait depuis Aubry c. Vice-Versa, [1998] 1 R.C.S. 591 qu'il faut «parler de violation du droit à l'image et, par conséquent, de faute dès que l'image est publiée sans consentement et qu'elle permet d'identifier la personne en cause», de même qu'on sait depuis Gauthier c. Beaumont, [1998] 2 R.C.S. 3, qu'un état psychologique de crainte peut constituer une cause d'impossibilité d'agir au sens de l'art. 2232 C.c.B.-C., et suspendre la prescription, lorsque cette crainte est causée par la faute du défendeur, etc... Même si, de l'intérieur du droit positif, ces propositions paraissent apodictiques, elles demeurent fragiles. Elles laissent toujours place à une qualification différente des faits (comme le démontrent par exemple le jugement dissident dans l'affaire Gauthier, ou encore les arrêts R. c. Askov, [1990] 2 R.C.S. 1199 et R. c. Morin, [1992] 1 R.C.S. 771) et, en l'absence de fondement constitutionnel, elles sont à la merci d'une intervention législative (comme le démontre, par exemple, un jugement comme Maison L'Intégrale c. Tribunal du travail, [1996] R.J.Q. 859 (C.A.)). 
à ceux qui s'adressent à eux pour obtenir justice, modérons leurs attentes. Et perpétuons les apparences : encore aujourd'hui, le juge «applique le droit», un point c'est tout. Le reste, comme disait Montaigne, n'est que «disceptation et agitation des diverses et contraires ratiocinations que la matière du droit souffre.» Le public n'en a rien à faire. 